\title{
A comparative study of bacterial diversity based on effects of three different shade shed types in the rhizosphere of Panax quiquefolium $\mathrm{L}$.
}

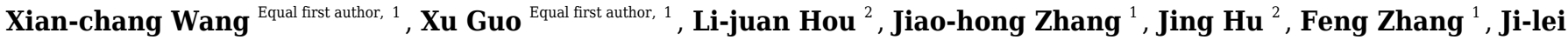 \\ Mao $^{2}$, Zhi-fen Wang ${ }^{1}$, Cong-jing Zhang ${ }^{1}$, Jin-long Han ${ }^{1}$, Yan-wei Zhu ${ }^{1}$, Chao Liu ${ }^{1}$, Jin-yue Sun ${ }^{1}$, Cheng-gang \\ Shan ${ }^{\text {Corresp. } 1}$ \\ ${ }^{1}$ Key Laboratory of Novel Food Resources Processing, Ministry of Agriculture and Rural Affairs/Research Center of Medicinal Plant, Shandong Academy of \\ Agricultural Sciences/Institute of Agro-Food Science and Technology, Shandong Academy of Agricultural Scie, Jinan, China \\ ${ }^{2}$ Weihai Academy of Agricultural Sciences, Weihai, China \\ Corresponding Author: Cheng-gang Shan \\ Email address: shanchenggang@126.com
}

Background. Shading is an important factor affecting the cultivation of American ginseng, as it influences crop quality and yield. Rhizosphere microorganisms are also crucial for normal plant growth and development. However, whether different shade types significantly change American ginseng rhizosphere microorganisms is unknown.

Methods. This study evaluated the rhizosphere soils of American ginseng under traditional, high flag and high arch shade sheds. High-throughput 16S rRNA gene sequencing determined the change of rhizosphere bacterial communities.

Results. The microbial diversity in rhizosphere soils of American ginseng significantly changed under different shading conditions. The bacteria diversity was more abundant in the high arch shade than flat and traditional shades. Different bacterial genera, including Bradyrhizobium, Rhizobium, Sphingomonas, Streptomyces and Nitrospira, showed significantly different abundances. Different shading conditions changed the microbial metabolic function in the American ginseng rhizosphere soils. The three types of shade sheds had specific enriched functional groups. The abundance of ATP-binding cassette (ABC) transporters consistently increased in the bacterial microbiota. These results help understand the influence of shading systems on the rhizosphere microecology of American ginseng, and contribute to the American ginseng cultivation. 


\section{A comparative study of bacterial diversity based on effects \\ 2 of three different shade shed types in the rhizosphere of \\ 3 Panax quiquefolium $\mathrm{L}$.}

4

5 Xianchang Wang ${ }^{1, *}, \mathrm{Xu} \mathrm{Guo}^{1, *}$, Lijuan $\mathrm{Hou}^{2}$, Jiaohong Zhang ${ }^{1}$, Jing $\mathrm{Hu}^{2}$, Feng Zhang ${ }^{1}$, Jilei

$6 \mathrm{Mao}^{2}$, Zhifen Wang ${ }^{1}$, Congjing Zhang ${ }^{1}$, Jinlong Han ${ }^{1}$, Yanwei Zhu ${ }^{1}$, Chao Liu ${ }^{1}$, Jinyue Sun ${ }^{1}$,

7 Chenggang Shan ${ }^{1}$

8

$9 \quad{ }^{1}$ Key Laboratory of Novel Food Resources Processing, Ministry of Agriculture and Rural

10 Affairs/Research Center of Medicinal Plant, Shandong Academy of Agricultural

11 Sciences/Institute of Agro-Food Science and Technology, Shandong Academy of Agricultural

12 Sciences, Jinan, Shandong, PR China

$13{ }^{2}$ Weihai Academy of Agricultural Sciences, Weihai, Shandong, PR China

* These authors contributed equally to this work.

17 Corresponding Author:

18 Chenggang Shan ${ }^{1}$

19202 Gongye North Road, Jinan, Shandong, 250100, PR China

20 Email address: shanchenggang@,126.com 


\section{Abstract}

22 Background. Shading is an important factor affecting the cultivation of American ginseng, as it influences crop quality and yield. Rhizosphere microorganisms are also crucial for normal plant growth and development. However, whether different shade types significantly change American ginseng rhizosphere microorganisms is unknown.

Methods. This study evaluated the rhizosphere soils of American ginseng under traditional, high flag and high arch shade sheds. High-throughput 16S rRNA gene sequencing determined the change of rhizosphere bacterial communities.

Results. The microbial diversity in rhizosphere soils of American ginseng significantly changed under different shading conditions. The bacteria diversity was more abundant in the high arch shade than flat and traditional shades. Different bacterial genera, including Bradyrhizobium, Rhizobium, Sphingomonas, Streptomyces and Nitrospira, showed significantly different abundances. Different shading conditions changed the microbial metabolic function in the American ginseng rhizosphere soils. The three types of shade sheds had specific enriched functional groups. The abundance of ATP-binding cassette (ABC) transporters consistently increased in the bacterial microbiota. These results help understand the influence of shading systems on the rhizosphere microecology of American ginseng, and contribute to the American ginseng cultivation. 


\section{Introduction}

American ginseng (Panax quinquefolius L.) is a perennial understory herb of the Araliaceae family (Cruse-Sanders \& Hamrick 2004). The herb improves the overall health of human beings by boosting vitality, improving the immune system and protecting against stress. American ginseng is also a traditional medicine for several pharmacological functions, including antiinflammatory, anti-cancer activities, anti-diabetes, obesity treatment, and enhancing the cardio cerebral vascular, and central nervous systems (Izzo 2009; Li et al. 2010; Poddar et al. 2011; Tsao \& Liu 2007; Wang et al. 2007). This herb originated from the eastern part of North America (Nadeau \& Olivier 2003) and reached China in the 1980s (Qin et al. 2018). Today, the American ginseng planting area in northern China exceeds 10,000 ha (Jiao et al. 2019).

In native areas, American ginseng grows as an understory plant in deciduous and mixed forests (Punja 2011), preferring mild and humid climates. The latitude at the origin of American ginseng is similar to vast areas in China, but the rainfall patterns and altitudes vary. This herb prefers higher air humidity with well ventilated, loose soils, suitable for water supply without waterlogging. The optimal growth temperature of American ginseng is $10-28^{\circ} \mathrm{C}$ (Nadeau \& Olivier 2003). As a typical shade plant, American ginseng prefers oblique, scattered lights. The direct sunlight around noon inhibits photosynthesis, cause photobleaching and leaf death (Proctor \& Palmer 2017). Therefore, artificial shades are necessary for American ginseng plantations to mimic natural planting conditions (Hongpeng et al. 2018). There are various shade types according to permeability and height. By permeability, the types included, total shade, single and double transparent shades. By height, there are high and low shades. The roof structures may be arch, slope, and flat, constructed using straw cover, wood board, cloth, reed curtain and sunshade net. Shade supporting materials include wood, metal, cement and bamboo pole. The shade structure is classified as simplex and duplex structures, according to the structural complexity. Shades influence the American ginseng soil rhizosphere.

The rhizosphere is the narrow soil region that directly contacts plant roots and facilitates inorganic and organic matter exchange between roots and the soil. This rhizosphere exchange is key for normal plant growth and development (Broeckling et al. 2008; Schmidt et al. 2019). Plants often alter the rhizosphere for adaptation to changing environments (Ryan et al. 2001). The rhizosphere harbors numerous microorganisms that constitute a complex community of plant-associated microorganisms important for plant health (Berendsen et al. 2012). Microorganisms are essential for plant productivity and health, but some cause plant diseases (Chisholm et al. 2006). Plant-associated microorganisms, known as the second genome of plants, have recently attracted extensive research (Berg et al. 2014; Turner et al. 2013). American ginseng cultivation is presently the main development agenda, and shading treatment is the 
decisive factor for ginseng growth and development. Shading determines the economic and biological yield of this vital herb. However, whether or not different shade types change the rhizosphere microorganisms of American ginseng is unknown.

This research analyzed the $16 \mathrm{~S}$ rRNA bacterial sequences of the American ginseng root rhizosphere to estimate bacterial diversity under three different shade types. The rhizosphere soil has numerous bacterial genera, but the rhizosphere bacteria abundance under the three vary shades. The study results help understand the effects of shading systems on rhizosphere microecology for American ginseng cultivation.

\section{Materials \& Methods}

\section{General situation of this experiment}

The experiment was performed in the Weihai farmland ginseng experimental area of Shandong Province $\left(122^{\circ} 27^{\prime}\right.$ E, $36^{\circ} 96^{\prime}$ N). Weihai is among the leading American ginseng production areas of China. As a shade-loving plant, American ginseng must be cultivated under shading conditions. Therefore, farmers began to use shade shed to ensure the yield and quality of American ginseng. This study adopted traditional (CTP), high flag (PP), and high arch shade sheds (GP) (Fig. 1). The traditional shed is a flat, wooden structure of $1.6 \mathrm{~m}$, height. The high flat shed is a flat alloy structure, $2.2 \mathrm{~m}$ in height, while the high arch shed is an alloy structure measuring $2.6 \mathrm{~m}$ in height. All three sheds have double-layer shading nets.

Basal fertilizer $(\sim 3-4 \mathrm{t} / \mathrm{mu})$ was applied once before the experimentation, conducted between 2016 and 2018. The shading shed was a completely random block with three replicates.

\section{Soil collection}

At the end of October 2018 when 3-year-old ginseng roots were manually harvested, 0-10 $\mathrm{cm}$ of surface soil was randomly sampled. Six soil samples from one type of shed were combined to form one sample. Each shed type had three composite samples, namely CTP1, CTP2, CTP3, PP1, PP2, PP3, GP1, GP2 and GP3. One part of each sample was stored at $-80^{\circ} \mathrm{C}$ for subsequent DNA extraction. The other portion was homogenized and sieved with a $2-\mathrm{mm}$ mesh sieve to remove large particles, air-dried and used for chemical analysis. Soil $\mathrm{pH}$ was determined at 1:2 soil: water ratio using a combination glass electrode (FE20K, Mettler-Toledo, Swiss). Soil total organic matter (TOM) was assayed following Walkley-Black method. Hydrolyzable nitrogen $(\mathrm{HN})$ and available phosphorus (AP) were determined by chemical analytical methods, as described by Liu et al.(Liu et al. 2014) and Murphy et al. (Murphy \& Riley 1962). The chemical characteristics of these soil samples are shown in Table S1. The yield of American ginseng, disease incidence, type and severity are shown in Table S2. The disease severity index was calculated using the method described by Chiang et al (Chiang et al. 2017). 


\section{DNA extraction, PCR amplification and sequencing}

Total DNA was extracted using the Z.N.ATM Mag-Bind Soil DNA Kit (Omega Bio-Tek, GA, USA). DNA was separated and visually tested for quality on a $0.8 \%(\mathrm{w} / \mathrm{v})$ agarose gel electrophoresis. The DNA concentration was measured with a NanoDrop ${ }^{\mathrm{TM}} 2000$ spectrophotometer (Thermo Fisher Scientific, MA, USA). DNA extracts were preserved at $-20^{\circ} \mathrm{C}$ for PCR.

The bacterial amplicon library was obtained using the 338F (5'ACTCCTACGGGAGGCAGCA-3') (Wu et al. 2016) and 806R (5'GGACTACHVGGGTWTCTAAT-3') (Wu et al. 2016) primers, which target the V3-V4 region of the 16S rRNA gene. Personalbio Technology (Shanghai, China) generated 300 bp paired-end reads from the qualified libraries using an Illumina MiSeq platform (Illumina, CA, USA) following the manufacturer's instructions. The raw reads were deposited to the NCBI Sequence Read Archive under accession number PRJNA662686.

\section{Sequencing data analysis, OTU production and annotation}

Split reads were sorted into each sample by their unique barcodes using QIIME V.1.8.0 (Bokulich et al. 2013; Caporaso et al. 2010). The sequencing barcodes and primer sequences were cut off from original paired-end reads using the FLASH V1.2.7 (Magoč \& Salzberg 2011). The USEARCH software (v5.2.236, http://www.drive5.com/usearch/) was used to detect and remove chimera sequences.

The effective tags with $\geq 97 \%$ similarity were assigned to the same operational taxonomic units (OTUs) using the Uparse software (v8.1.1861). The sequence with the highest occurrence frequency in each OTU was considered the representative sequence for further annotation (Edgar 2013). Each representative sequence was annotated using the UCLUST method (Edgar 2010) on the Greengenes database (Release 13.8, http://greengenes.secondgenome.com/) (DeSantis et al. 2006). the annotation levels were: kingdom, phylum, class, order, family, genus and species to determine the community composition of each sample. Rare OTUs (singletons to tripletons), potentially originating from artificial sequences, were removed. The read counts were normalized considering the smallest read number per sample.

\section{Statistical analysis}

All statistical analyses were calculated using the QIIME software (Version 1.8.0) and displayed using the R software (Version 2.15.3). All data were analyzed using a one-way analysis of variance (ANOVA) (P value $<0.05$ ) with the SPSS19.0 software (SPSS Inc., Chicago, USA). The differences between groups were compared using Fisher's least significant difference test.

Alpha diversity was applied to analyze the complexity of species diversity for each community through four indices, including abundance-based coverage (ACE) estimator, Chao 1, 
144

145

146

147

148

149

150

151

152

153

154

155

156

157

158

159

160

161

162

163

164

165

166

167

168

169

170

171

172

173

174

175

176

177

178

Shannon diversity and Inverse Simpson indices. The ACE estimator and Chao 1 index determined the community richness, while the Shannon and Inverse Simpson indices estimated the community diversity. All samples were randomly resampled at the lowest sequencing depth level of $90 \%$ to correct the diversity difference caused by sequencing depth. Then, QIIME software is used to calculate alpha diversity.

The principal component analysis (PCA) determined the beta diversity to reduce the dimension of the original variables. Hierarchical clustering was performed using the Unweighted Pair-Group Method with Arithmetic Means (UPGMA) clustering to interpret the distance matrix using average linkage.

The Spearman's rank correlation analysis performed using the Mothur software (Schloss et al. 2009). The Cytoscape tool (http://www.cytoscape.org/) displayed the first 50 correlated groups. The PICRUSt tool

(http://huttenhower.sph.harvard.edu/galaxy/tool_runner?tool_id=PICRUSt_normalize) determined the enriched pathways following the Kyoto Encyclopedia of Genes and Genomes (KEGG) of the microbiota. The variation analysis was performed following the functional abundance of the samples.

The SPSS 19.0 software determined the Spearman's rank correlation analysis between the microbial community composition and soil physicochemical parameters. Meanwhile, the Redundancy analysis (RDA) was performed using the Canoco 5.0 tool to identify the relationships between the bacterial community structures and soil physicochemical parameters.

\section{Results}

\section{Sequencing statistic}

A total of 660,115 raw tags were generated from 16S rRNA genes of the V3-V4 region. After trimming and filtering, 430,843 high-quality reads were obtained, with a 415 bp average reading length. The OTU clustering ( $97 \%$ similarity) generated 33,858 OTUs from the nine samples, with $99.97 \%$ classified under phylum. The rarefaction curves indicated that the sampling sufficiently captured the overall composition of each sample (Fig. S3).

\section{Taxonomic composition of bacterial assemblages in rhizosphere soil}

The taxonomy of 16S rRNA gene amplicons and the relative abundance of the top twenty taxa estimated the bacterial assemblages and variability in American ginseng rhizosphere soils from three different shade sheds (Fig. S4). At the phyla level, Proteobacteria was the most prominent taxon (30.5\%) of the total bacterial community, followed by Chloroflexi (17.2\%), Acidobacteria (15.4\%), Actinobacteria (15.3\%), and Gemmatimonadetes (5.8\%) (Fig. S4A). The dominant classes with $>1 \%$ relative abundance across all samples included Alphaproteobacteria, Acidobacteriia, Gammaproteobacteria, KD4-96, Gemmatimonadetes, Bacteroidia, 
179

180

181

182

183

184

185

186

187

188

189

190

191

192

193

194

195

196

197

198

199

200

201

202

203

204

205

206

207

208

209

210

211

212

213

214

Actinobacteria, Thermoleophilia, Deltaproteobacteria, Subgroup_6, Saccharimonadia, Ktedonobacteria, Anaerolineae, Acidimicrobiia, Clostridia, AD3, Verrucomicrobiae, Planctomycetacia, Blastocatellia (Subgroup_4), and Parcubacteria (Fig. S4B). Bacterial variation increased from phylum to genus, following analysis of the ten most-abundant classes at the order, family and genus levels (Fig. S4C-S4E).

\section{Alpha and beta diversity analysis of samples}

The ACE, Chao 1, Shannon diversity, and Inverse Simpson indices quantified the alpha diversity and per sample bacterial diversities. The ACE and Chao 1 in PP were significantly lower $(\mathrm{P}<0.05)$ than GP and CTP (Fig. 2), demonstrating that shading affected the rhizosphere bacterial richness. The Shannon diversity and Inverse Simpson indices showed no significant differences in bacterial diversity among the three shade sheds.

At the OTU level, PC1 explained $60.77 \%$ and PC2 $13.07 \%$ of the total bacterial variation, clearly distinguishing the community structure of soil bacteria under the three shade sheds (Fig. 3A). All the samples collected from the rhizosphere were distinguishable based on the UPGMAgenerated cluster trees (Fig. 3B).

Variations in the bacterial community of American ginseng rhizosphere soil under three different shade sheds

The least discriminant analysis (LDA) effect size taxonomic cladogram compared the phylum to the genus in the three sample communities to expose the bacterial variation in the American ginseng rhizosphere soil under three different shade sheds. The relative abundances of bacterial phyla were different across the three shade shed rhizosphere soils (Fig. 4). The relative abundances of Proteobacteria, Nitrospirae and WS2 in GP were significantly higher than PP and CTP (LSD, $\mathrm{p}<0.05$ ). Dependentiae, Elusimcrobia, FCPU426 and WPS- 2 were significantly abundant in the pp than GP and CTP (LSD, $p<0.05)$. At a finer taxonomic level, the three shade sheds were significantly different in some bacterial genera. The abundance values of the 50 most abundant genera from the three shade sheds are shown in Fig. S5.

\section{Correlation and KEGG functional analysis in bacterial communities}

The relationships between abundance values of the various bacterial genera were determined following the abundance changes of different genera in the soil samples. The genera relationship network indicated a complex functional collaboration within the microbiota (Fig. S6). The KEGG functional enrichment analysis of bacterial microbiota from rhizosphere soils under the three different shade sheds determined their bacterial functional diversities. The abundance of the top 50 functional groups was analyzed and displayed on a heat-map (Fig. 5). A Venn diagram (Fig. 6) shows the number of OTUs of the common functional soil bacteria in the three shade sheds, indicating the functional divergence of bacterial microbiota in the rhizosphere of American ginseng roots. 
215

216

217

218

219

220

221

222

223

224

225

226

227

228

229

230

231

232

233

234

235

236

237

238

239

240

241

242

243

244

245

246

247

248

\section{Relationship between microbial communities and soil chemical properties}

The Spearman's rank correlation coefficient determined the relationship between soil microbial diversity and soil chemical properties (Fig.7). The Chao 1 and Shannon diversity indices positively and significantly correlated with the soil $\mathrm{pH}(\mathrm{P}<0.05)$. However, Chao 1 and ACE indices negatively correlated with soil TOM and AP contents $(\mathrm{P}<0.01)$, while the Inverse Simpson index negatively correlated with $\mathrm{HN}(\mathrm{P}<0.05)$.

RDA analysis was performed to observe the potential correlations between the community structures at genus levels and soil chemical properties in the GP, PP and CTP. The RDA analysis results showed that the first two RDA axes (RDA1 and RDA2) account for $60.30 \%$ and $12.67 \%$ of the variance, respectively (Fig. 8). Soil $\mathrm{pH}(\mathrm{F}=9.2, \mathrm{P}=0.016)$ was the most important contributor to bacterial community variation (Table S7).

\section{Discussion}

This study investigated the changes of American ginseng rhizosphere soil bacterial communities under three different shade sheds. The GP microbial diversity was higher than PP and CTP. Meanwhile, the soil physicochemical properties affected microbial diversity. It has been reported that the bacterial diversity of grapes rhizosphere changed under different environmental conditions (Bokulich et al. 2014). In this study, the soil physicochemical properties under the different shade sheds were different (Table S1). The pH of GP was significantly higher than PP and CTP, while the TOM and AP content of PP was higher than GP and CTP. pH is a key driving factor of the soil bacterial community, which determines the availability of soil nutrients (Lauber et al. 2009; Shi et al. 2015). Increasing the $\mathrm{pH}$ increased the soil bacteria diversity (Rousk et al. 2009). In this study, Spearman's correlation analysis showed that the pH positively and significantly correlated with the microbial diversity (Fig. 7).

Besides the above attributes, the most critical resource for bacterial growth is organic matter (Laganière et al. 2013), and nitrogen is the second limiting nutrient (Frey 2004). However, TOM, AP and HN negatively correlated with microbial diversity. The community structure of microorganisms varied under the three different shade sheds (Van der Heijden \& Wagg 2013). The soil physicochemical properties affected the microbial structure, shown by RDA analysis. However, $\mathrm{pH}$ was the key environmental factor affecting the composition of the microbial community (Fig. 8). The diversity of soil microorganisms is related to soil health and quality (Anderson 2003; Garbeva et al. 2004). Decreased soil microbial diversity causes soil-borne plant diseases while changing growth conditions increase the risks of phytopathogens (Burie et al. 2011; Pugliese et al. 2011).

This study reported varying incidences and severity of diseases affecting American ginseng 
249

250

251

252

253

254

255

256

257

258

259

260

261

262

263

264

265

266

267

268

269

270

271

272

273

274

275

276

277

278

279

280

281

282

283

under the three shade types. The average disease incidence of American ginseng under GP, PP and CTP were 3.58, 5.19, and 12.65\%, respectively. However, the average disease severity for GP, PP and CTP were 10.5, 9.8, and $18.6 \%$, respectively. The incidence and severity of American ginseng diseases under GP and PP were significantly lower than CTP. Interestingly, the fresh weight of American ginseng in GP and PP was higher than in CTP. Therefore, the type of shade is critical for disease control and the yield increase in American ginseng.

American ginseng is a perennial plant whose root exudates accumulate in the rhizosphere, providing substrates for several biological communities. Root exudates are considered a driving force for selecting specific microbial populations in the rhizosphere (Bais et al. 2006; Garbeva et al. 2004; Mazzola \& Manici 2012). For example, the proportion of Proteobacteria and Bacteroidetes increased because of their increased relative abundances in a high nitrogen area (Fierer et al. 2012). Gram-negative Proteobacteria are dominant in the three soil samples used in this study, causing legume symbiotic nitrogen fixation (Raymond et al. 2004). Bradyrhizobium, a gram-negative Proteobacteria, had the highest relative abundance in the rhizosphere soil of the American ginseng sampled in this study. Bradyrhizobium, genus Azotobacterthat, induces nodule formation in the roots of legumes (Long 1996). However, the American ginseng lacked root nodules, probably due to the absence of nod receptors or defects of subsequent kinase cascades in American ginseng, which are essential for legume nodule formation (Gage 2004; Smit et al. 2007). Sphingomonas was the second largest genus in the rhizosphere of American ginseng under the three shade types. Genus Sphingomonas commonly degrade various aromatic compounds (Fredrickson et al. 1995). Therefore, Sphingomonas accumulation in the rhizosphere indirectly implies the secretion of different aromatic secondary metabolites in American ginseng roots. Another abundant genus, Nitrospira species, is critical for the nitrogen cycle in water and soil (Bartosch et al. 2002). The content of both Sphingomonas and Nitrospira in the high arch shed soil was higher than the other two sheds. The study also identified that Gemmatimonas had high relative abundance. The Gemmatimonas acquire various plant processing resources, and their abundance negatively correlates with plant growth (H. et al. 2015).

As expected, the relative abundance of some microbial communities decreased due to specialized antimicrobial metabolites in the root exudates (Bais et al. 2006; Berg \& Smalla 2009; Mazzola \& Manici 2012). Additionally, changes in the chemical properties of soils probably changed the microbial community composition (Lauber et al. 2008). The analyses showed significantly different $\mathrm{pH}$, organic matter, hydrolytic nitrogen, and available phosphorus among the three different shade sheds (Table S1), but the effect mechanism on the microbial community needs further verification. Changes in the composition of bacterial communities may change metabolism, biodegradation and disease inhibition (Bell et al. 2013; Garbeva et al. 
284

285

286

287

288

289

290

291

292

293

294

295

296

297

298

299

300

301

302

303

304

305

306

307

308

309

310

311

312

313

314

315

316

317

2004). These results show that shade sheds improve soil productivity, and appropriate shade shads can modify soil microbial communities.

Considering the KEGG enrichment analysis, half of the 50 most abundant functional groups in the microbial community of the three shade types are transporters (Fig.5). The other half involved metabolism, such as amino acid (e.g. k00384, k01704, k01703), fatty acid (e.g. k00626, k00059, k01897) and secondary metabolism (e.g. k01952, k01834, k01692). Among the 26 transporters, 22 are ATP-binding cassette $(\mathrm{ABC})$ transporters. ABC transporters are integral membrane proteins that combine substrate trans lipid bilayers transport with ATP hydrolysis (Hollenstein et al. 2007). ABC transporters are essential for the survival of bacteria as they catalyze nutrient uptake by bacteria and facilitate the efflux of toxic or antibacterial drugs. ABC transporters also export various virulence factors, including antibiotics, bacteriocins, and toxins, increasing within-community competition and hindering pathogen invasion (Davidson \& Chen 2004). The accumulation of bacterial rich in ABC transporters in the rhizosphere mediates inorganic and organic matter exchange between the root and soil (Ryan et al. 2001). CTP had the most abundant expression of ABC transporters (Fig.5), probably because CTP had the highest pathogenic rate and required more $\mathrm{ABC}$ transporters for defense against pathogen infection. The structure and composition of microbial communities are closely related to their metabolic function. This study identified 5625 functional groups in the three shade types, including 15 specific functional groups for the flat shed, 23 for the traditional shed, and 58 for the high arch shed (Fig.6). The high arch shade sheds had a significantly higher number of specific functional groups than the other two sheds.

\section{Conclusions}

The diversity of American ginseng soil microbial community structure and function changed under the three different shade sheds. Shade sheds improve soil productivity, an effective defense mechanism for American ginseng. Shade sheds also balance soil microbial communities, and shading enriches the metabolic functions of soil microorganisms. This work provides a theoretical understanding of shading effect on the rhizosphere microecology of American ginseng.

\section{Acknowledgement}

This study was supported by Agricultural Science and Technology Innovation Project of Shandong Academy of Agricultural Sciences, China (NO. CXGC2021A50, CXGC2021A18); the National Natural Science Foundation of China (No. 82003632); the Provincial Major Scientific and Technological Innovation Project of Shandong, China (NO. 2019JZZY020612); the Province Agricultural Major Application Technology Innovation Project of Shandong, China 
318 (NO. SD2019ZZ016); the Provincial Key Research and Development Program of Shandong, 319 China (NO. 2019LYXZ025, 2019GSF109087); the Taishan Scholars's Program of Shandong for 320 Jinyue Sun.

\section{References}

Anderson T-H. 2003. Microbial eco-physiological indicators to asses soil quality. Agriculture, Ecosystems \& Environment 98:285-293 DOI 10.1016/S0167-8809(03)00088-4.

Bais HP, Weir TL, Perry LG, Gilroy S, and Vivanco JM. 2006. The role of root exudates in rhizosphere interactions with plants and other organisms. Annual Review of Plant Biology 57:233-266 DOI 10.1146/annurev.arplant.57.032905.105159.

Bartosch S, Hartwig C, Spieck E, and Bock E. 2002. Immunological detection of Nitrospira-like bacteria in various soils. Microbial Ecology 43:26-33 DOI 10.1007/s00248-001-0037-5.

Bell TH, Yergeau E, Maynard C, Juck D, Whyte LG, and Greer CW. 2013. Predictable bacterial composition and hydrocarbon degradation in Arctic soils following diesel and nutrient disturbance. ISME Journal 7:12001210 DOI 10.1038/ismej.2013.1.

Berendsen RL, Pieterse CM, and Bakker PA. 2012. The rhizosphere microbiome and plant health. Trends in Plant Science 17:478-486 DOI 10.1016/j.tplants.2012.04.001.

Berg G, Grube M, Schloter M, and Smalla K. 2014. Unraveling the plant microbiome: looking back and future perspectives. Frontiers in Microbiology 5:148 DOI 10.3389/fmicb.2014.00148.

Berg G, and Smalla K. 2009. Plant species and soil type cooperatively shape the structure and function of microbial communities in the rhizosphere. FEMS Microbiology Ecology 68:1-13 DOI 10.1111/j.15746941.2009.00654.x.

Bokulich NA, Subramanian S, Faith JJ, Gevers D, Gordon JI, Knight R, Mills DA, and Caporaso JG. 2013. Qualityfiltering vastly improves diversity estimates from Illumina amplicon sequencing. Nature Methods 10:57-59 DOI 10.1038/nmeth.2276.

Bokulich NA, Thorngate JH, Richardson PM, and Mills DA. 2014. Microbial biogeography of wine grapes is conditioned by cultivar, vintage, and climate. Proceedings of the National Academy of Sciences of the United States of America 111:E139-148 10.1073/pnas.1317377110.

Bremmer JM, and Mulvaney CS. 1982. Total nitrogen. In: Methods of soil analysis, part 2 Chemical and microbiological properties, 2nd edn. (eds Page, A. L., Miller, R. H. \& Keeney, D. R.). American Society of Agronomy, Madison: 595-624 
Broeckling CD, Broz AK, Bergelson J, Manter DK, and Vivanco JM. 2008. Root exudates regulate soil fungal community composition and diversity. Applied and Environmental Microbiology 74:738-744 DOI 10.1128/aem.02188-07.

Burie JB, Langlais M, and Calonnec A. 2011. Switching from a mechanistic model to a continuous model to study at different scales the effect of vine growth on the dynamic of a powdery mildew epidemic. Annals of Botany 107:885-895 DOI 10.1093/aob/mcq233.

Caporaso JG, Kuczynski J, Stombaugh J, Bittinger K, Bushman FD, Costello EK, Fierer N, Peña AG, Goodrich JK, Gordon JI, Huttley GA, Kelley ST, Knights D, Koenig JE, Ley RE, Lozupone CA, McDonald D, Muegge BD, Pirrung M, Reeder J, Sevinsky JR, Turnbaugh PJ, Walters WA, Widmann J, Yatsunenko T, Zaneveld J, and Knight R. 2010. QIIME allows analysis of high-throughput community sequencing data. Nature Methods 7:335-336 DOI 10.1038/nmeth.f.303.

Chiang K-S, Liu HI, Tsai JW, Tsai JR, and Bock C. 2017. A discussion on disease severity index values. Part II: using the disease severity index for null hypothesis testing. Annals of Applied Biology 171:490-505 10.1111/aab.12396.

Chisholm ST, Coaker G, Day B, and Staskawicz BJ. 2006. Host-microbe interactions: shaping the evolution of the plant immune response. Cell 124:803-814 DOI 10.1016/j.cell.2006.02.008.

Cruse-Sanders JM, and Hamrick JL. 2004. Genetic diversity in harvested and protected populations of wild American ginseng, Panax quinquefolius L. (Araliaceae). American Journal of Botany 91:540-548 DOI 10.3732/ajb.91.4.540.

Davidson AL, and Chen J. 2004. ATP-binding cassette transporters in bacteria. Annual Review of Biochemistry 73:241-268 DOI 10.1146/annurev.biochem.73.011303.073626.

DeSantis TZ, Hugenholtz P, Larsen N, Rojas M, Brodie EL, Keller K, Huber T, Dalevi D, Hu P, and Andersen GL. 2006. Greengenes, a chimera-checked $16 \mathrm{~S}$ rRNA gene database and workbench compatible with ARB. Applied and Environmental Microbiology 72:5069-5072 DOI 10.1128/aem.03006-05.

Edgar RC. 2010. Search and clustering orders of magnitude faster than BLAST. Bioinformatics 26:2460-2461 10.1093/bioinformatics/btq461.

Edgar RC. 2013. UPARSE: highly accurate OTU sequences from microbial amplicon reads. Nature Methods 10:996998 DOI 10.1038/nmeth.2604.

Fierer N, Lauber CL, Ramirez KS, Zaneveld J, Bradford MA, and Knight R. 2012. Comparative metagenomic, phylogenetic and physiological analyses of soil microbial communities across nitrogen gradients. ISME Journal 6:1007-1017 DOI 10.1038/ismej.2011.159. 
Fredrickson JK, Balkwill DL, Drake GR, Romine MF, Ringelberg DB, and White DC. 1995. Aromatic-degrading Sphingomonas isolates from the deep subsurface. Applied and Environmental Microbiology 61:1917-1922 DOI 10.1128/aem.61.5.1917-1922.1995.

Frey S. 2004. Chronic nitrogen enrichment affects the structure and function of the soil microbial community in temperate hardwood and pine forests. Forest Ecology and Management 196 10.1016/S03781127(04)00198-7.

Gage DJ. 2004. Infection and invasion of roots by symbiotic, nitrogen-fixing rhizobia during nodulation of temperate legumes. Microbiology and Molecular Biology Reviews 68:280-300 DOI 10.1128/mmbr.68.2.280-300.2004.

Garbeva P, van Veen JA, and van Elsas JD. 2004. Microbial diversity in soil: selection microbial populations by plant and soil type and implications for disease suppressiveness. Annual Review of Phytopathology 42:243-270 DOI 10.1146/annurev.phyto.42.012604.135455.

H. I, Franke-Whittle, Manici LM, and Heribert Insam BS. 2015. Rhizosphere bacteria and fungi associated with plant growth in soils of three replanted apple orchards. Plant and Soil 395:317-333 DOI 10.1007/s11104-015$2562-x$

Hollenstein K, Dawson RJ, and Locher KP. 2007. Structure and mechanism of ABC transporter proteins. Current Opinion in Structural Biology 17:412-418 DOI 10.1016/j.sbi.2007.07.003.

Hongpeng LI, Yingjiao LI, Zhao Y, and Xiangguo LI. 2018. Effects of Different Shading Treatments on Agronomic Characters of Cultivated Ginseng (Panax ginseng) in Farmland. Agricultural Biotechnology v.7:146-149+153 DOI 10.19759/j.cnki.2164-4993.2018.02.034.

Izzo AA. 2009. Phase II clinical trial reveals the excellent safety profile of Panax quinquefolius (American ginseng). Focus on Alternative and Complementary Therapies 14:24-25 DOI 10.1111/j.2042-7166.2009.tb01862.x.

Jiao XL, Zhang XS, Lu XH, Qin R, Bi YM, and Gao WW. 2019. Effects of maize rotation on the physicochemical properties and microbial communities of American ginseng cultivated soil. Scientific Reports 9:8615 DOI 10.1038/s41598-019-44530-7.

Laganière J, Paré D, Bergeron Y, Brassard B, and Cavard X. 2013. Stability of Soil Carbon Stocks Varies with Forest Composition in the Canadian Boreal Biome. Ecosystems 16:852-865 10.1007/s10021-013-9658-z.

Lauber C, Hamady M, Knight R, and Fierer N. 2009. Pyrosequencing-Based Assessment of Soil pH as a Predictor of Soil Bacterial Community Structure at the Continental Scale. Applied and environmental microbiology 75:5111-5120 10.1128/AEM.00335-09.

Lauber CL, Strickland MS, Bradford MA, and Fierer N. 2008. The influence of soil properties on the structure of bacterial and fungal communities across land-use types. Soil Biology and Biochemistry 40:2407-2415 DOI 
411

412

413

414

415

416

417

418

419

420

421

422

423

424

425

426

427

428

429

430

431

432

433

434

435

436

437

438

Li B, Wang CZ, He TC, Yuan CS, and Du W. 2010. Antioxidants potentiate American ginseng-induced killing of colorectal cancer cells. Cancer Letter 289:62-70 DOI 10.1016/j.canlet.2009.08.002.

Liu Z, Zhou W, Shen J, He P, Lei Q, and Liang G. 2014. A simple assessment on spatial variability of rice yield and selected soil chemical properties of paddy fields in South China. Geoderma 235-236:39-47 https://doi.org/10.1016/i.geoderma.2014.06.027.

Long SR. 1996. Rhizobium symbiosis: nod factors in perspective. Plant Cell 8:1885-1898 DOI 10.1105/tpc.8.10.1885.

Magoč T, and Salzberg SL. 2011. FLASH: fast length adjustment of short reads to improve genome assemblies. Bioinformatics 27:2957-2963 DOI 10.1093/bioinformatics/btr507.

Mazzola M, and Manici LM. 2012. Apple replant disease: role of microbial ecology in cause and control. Annual Review of Phytopathology 50:45-65 DOI 10.1146/annurev-phyto-081211-173005.

Murphy J, and Riley JP. 1962. A Modified Single Solution Method for the Dermination of Phosphate in Natural Waters. Anal Chim Acta 26:678-681

Nadeau I, and Olivier A. 2003. The biology and forest cultivation of American ginseng (Panax quinquefolius L.) in Canada. Canadian Journal of Plant Science 83:877-891

Poddar K, Kolge S, Bezman L, Mullin GE, and Cheskin L. 2011. Nutraceutical supplements for weight loss: a systematic review. Nutrition in Clinical Practice 26:539-552 DOI 10.1177/0884533611419859.

Proctor JTA, and Palmer JW. 2017. Optimal light for greenhouse culture of American ginseng seedlings. Journal of Ginseng Research 41:370-372 DOI 10.1016/j.jgr.2016.04.002.

Pugliese M, Gullino ML, and Garibaldi A. 2011. Effect of climate change on infection of grapevine by downy and powdery mildew under controlled environment. Communications in Agricultural \& Applied Biological Sciences 76:579-582

Punja ZK. 2011. American ginseng: research developments, opportunities, and challenges. Journal of Ginseng Research 35:368-374 DOI 10.5142/jgr.2011.35.3.368.

Qin Z, Jia C, Liao D, Chen X, and Li X. 2018. Comparison of Serum Metabolite Changes of Radiated Mice Administered with Panax quinquefolium from Different Cultivation Regions Using UPLC-Q/TOF-MS Based Metabolomic Approach. Molecules 23 DOI 10.3390/molecules23051014.

Raymond J, Siefert JL, Staples CR, and Blankenship RE. 2004. The natural history of nitrogen fixation. Molecular Biology and Evolution 21:541-554 DOI 10.1093/molbev/msh047.

Peer) reviewing PDF | (2020:12:56566:1:0:NEW 18 Nov 2021) 
439

440

441

442

443

444

445

446

447

448

449

450

451

452

453

454

455

456

457

458

459

460

461

462

463

464

465

466

467

468

469

Rousk J, Brookes P, and Bååth E. 2009. Contrasting Soil pH Effects on Fungal and Bacterial Growth Suggest Functional Redundancy in Carbon Mineralization. Applied and environmental microbiology 75:1589-1596 10.1128/AEM.02775-08.

Ryan P, Delhaize E, and Jones D. 2001. Function and mechanism of organic anion exudation from plant roots. Annual Review of Plant Physiology and Plant Molecular Biology 52:527-560 DOI 10.1146/annurev.arplant.52.1.527.

Schloss PD, Westcott SL, Ryabin T, Hall JR, Hartmann M, Hollister EB, Lesniewski RA, Oakley BB, Parks DH, Robinson CJ, Sahl JW, Stres B, Thallinger GG, Van Horn DJ, and Weber CF. 2009. Introducing mothur: open-source, platform-independent, community-supported software for describing and comparing microbial communities. Applied and Environmental Microbiology 75:7537-7541 DOI 10.1128/aem.01541-09.

Schmidt R, Ulanova D, Wick LY, Bode HB, and Garbeva P. 2019. Microbe-driven chemical ecology: past, present and future. ISME Journal 13:2656-2663 DOI 10.1038/s41396-019-0469-x.

Shi Y, Lou K, Li C, Wang L, Zhao Z-y, Zhao S, and Tian C-y. 2015. Illumina-based analysis of bacterial diversity related to halophytes Salicornia europaea and Sueada aralocaspica. Journal of Microbiology 53:678-685 10.1007/s12275-015-5080-x.

Smit P, Limpens E, Geurts R, Fedorova E, Dolgikh E, Gough C, and Bisseling T. 2007. Medicago LYK3, an entry receptor in rhizobial nodulation factor signaling. Plant Physiology 145:183-191 DOI 10.1104/pp.107.100495.

Tsao R, and Liu Z. 2007. Exploration and characterization of bioactive phytochemicals in native Canadian plants for human health. Canadian journal of plant science Revue canadienne de phytotechnie 87:1045-1053 DOI 10.4141/cjps07149.

Turner TR, James EK, and Poole PS. 2013. The plant microbiome. Genome Biology 14:209 DOI 10.1186/gb-2013-146-209.

Van der Heijden M, and Wagg C. 2013. Soil microbial diversity and agro-ecosystem functioning. Plant and Soil 363:15 10.1007/s11104-012-1545-4.

Wang CZ, Mehendale SR, and Yuan CS. 2007. Commonly used antioxidant botanicals: active constituents and their potential role in cardiovascular illness. American Journal of Chinese Medicine 35:543-558 DOI 10.1142/s0192415x07005053.

Wu X, Zhang H, Chen J, Shang S, Wei Q, Yan J, and Tu X. 2016. Comparison of the fecal microbiota of dholes highthroughput Illumina sequencing of the V3-V4 region of the 16S rRNA gene. Applied Microbiology and Biotechnology 100:3577-3586 DOI 10.1007/s00253-015-7257-y.

PeerJ reviewing PDF | (2020:12:56566:1:0:NEW 18 Nov 2021) 


\section{Figure 1}

Schematic drawings and photographs of the three different shade sheds.

(A) Schematic diagram of CTP. (B) A live shot inside the CTP. (C) A live shot of the exterior of the CTP. (D) Schematic diagram of PP. (E) A live shot inside the PP. (F) A live shot of the exterior of the PP. (G) Schematic diagram of GP. (H) A live shot inside the GP. (I) A live shot of the exterior of the GP.K

A

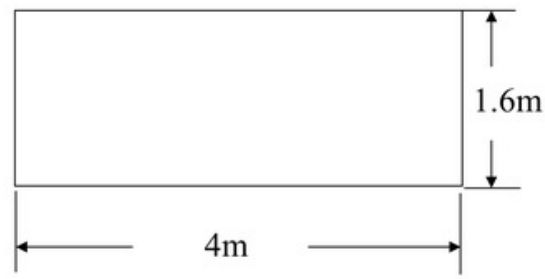

D

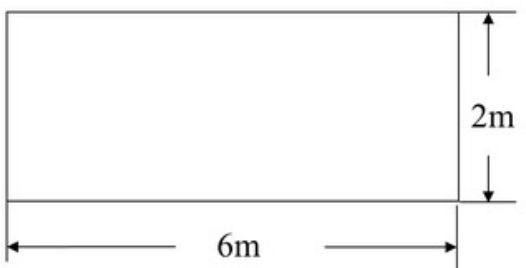

E
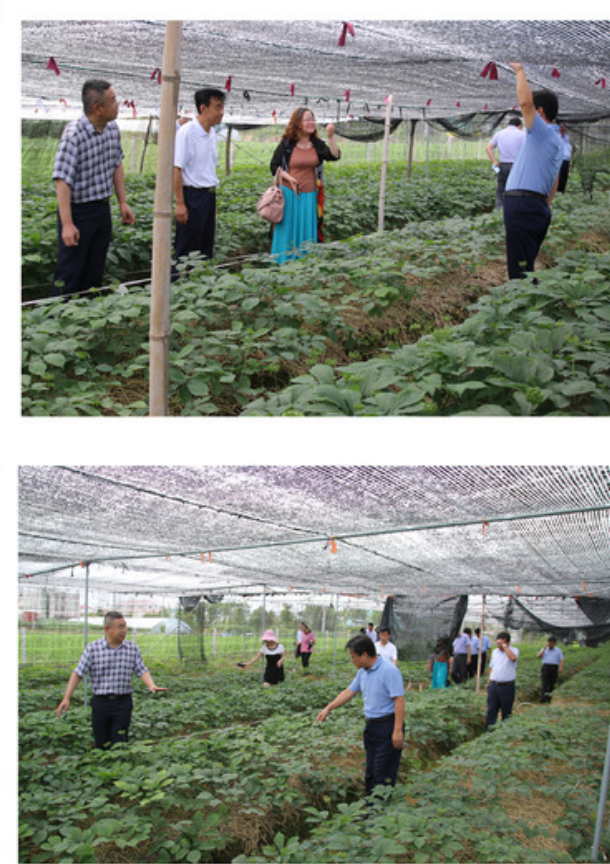

C

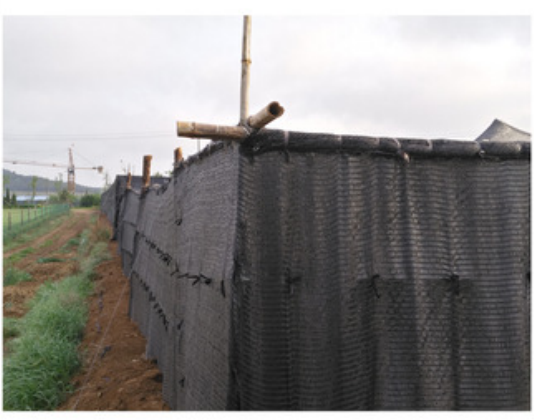

$\mathrm{F}$

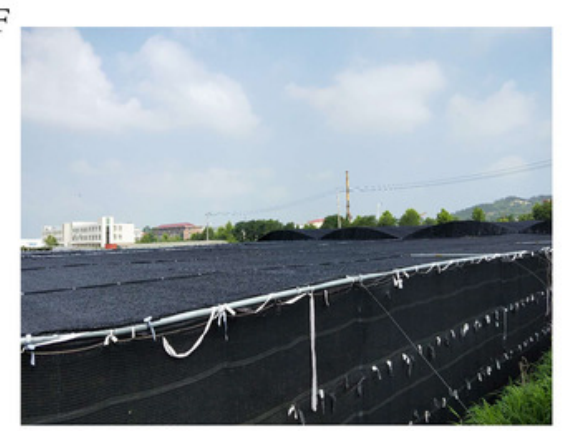

G

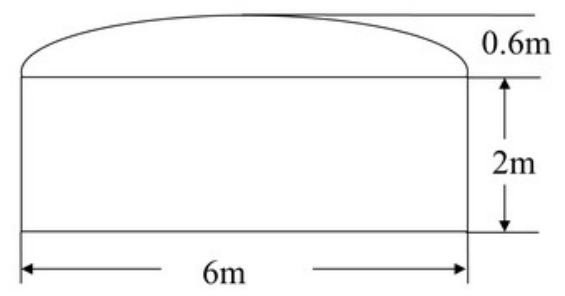

$\mathrm{H}$

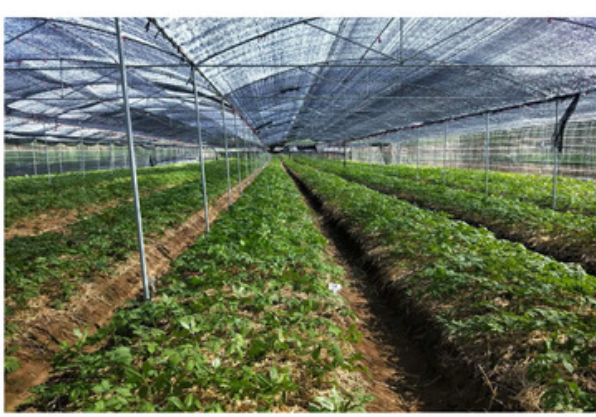

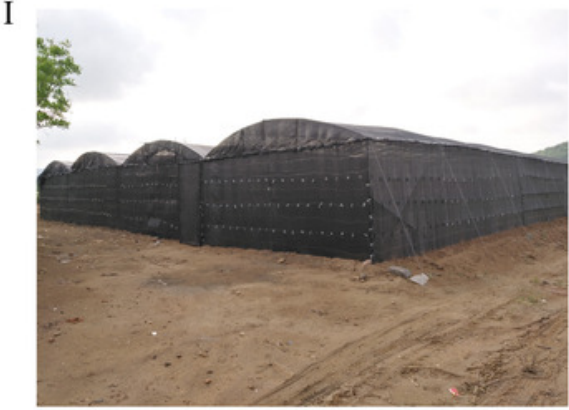


Figure 2

The Alpha diversity was estimated by Chao 1, ACE, Shannon index and Simpson in the rhizosphere soil under three shade sheds.

Significant changes determined by the T TEST. $\mathrm{P}<0.05$ are marked by an asterisk. $\mathrm{P}<0.01$ are marked by two asterisk. The unmarked are not significantly different.

A

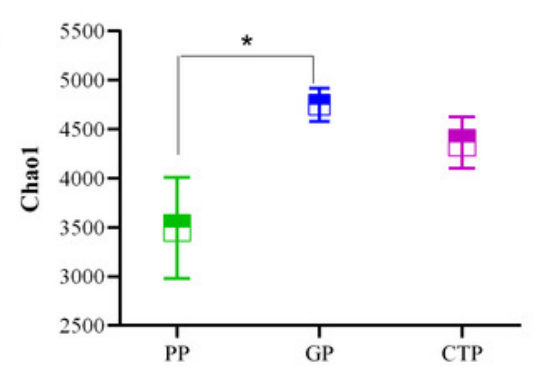

$\mathrm{C}$

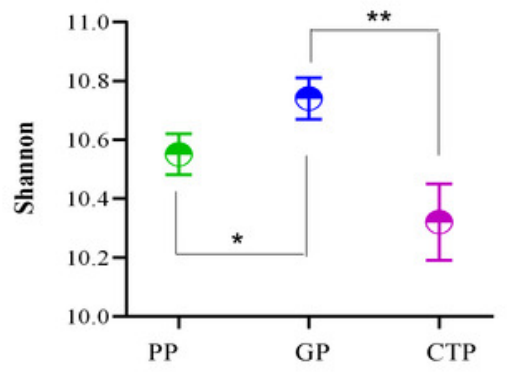

B

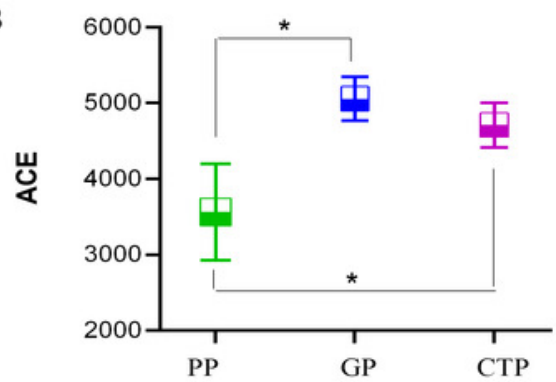

D

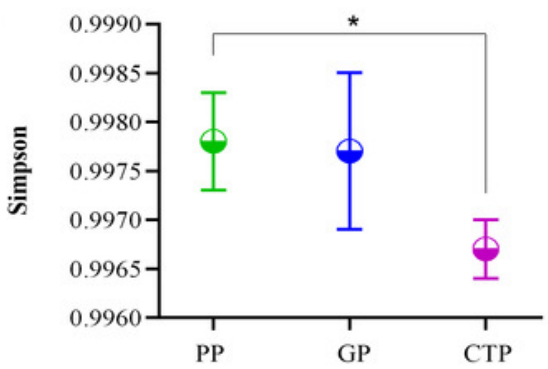


Figure 3

Beta diversity of the bacterial communities.

(A) PCA analysis of the soil bacterial communities based on the relative abundance of OTU.

(B) UPGMA cluster analysis based on Weighted UniFrac. . 
A

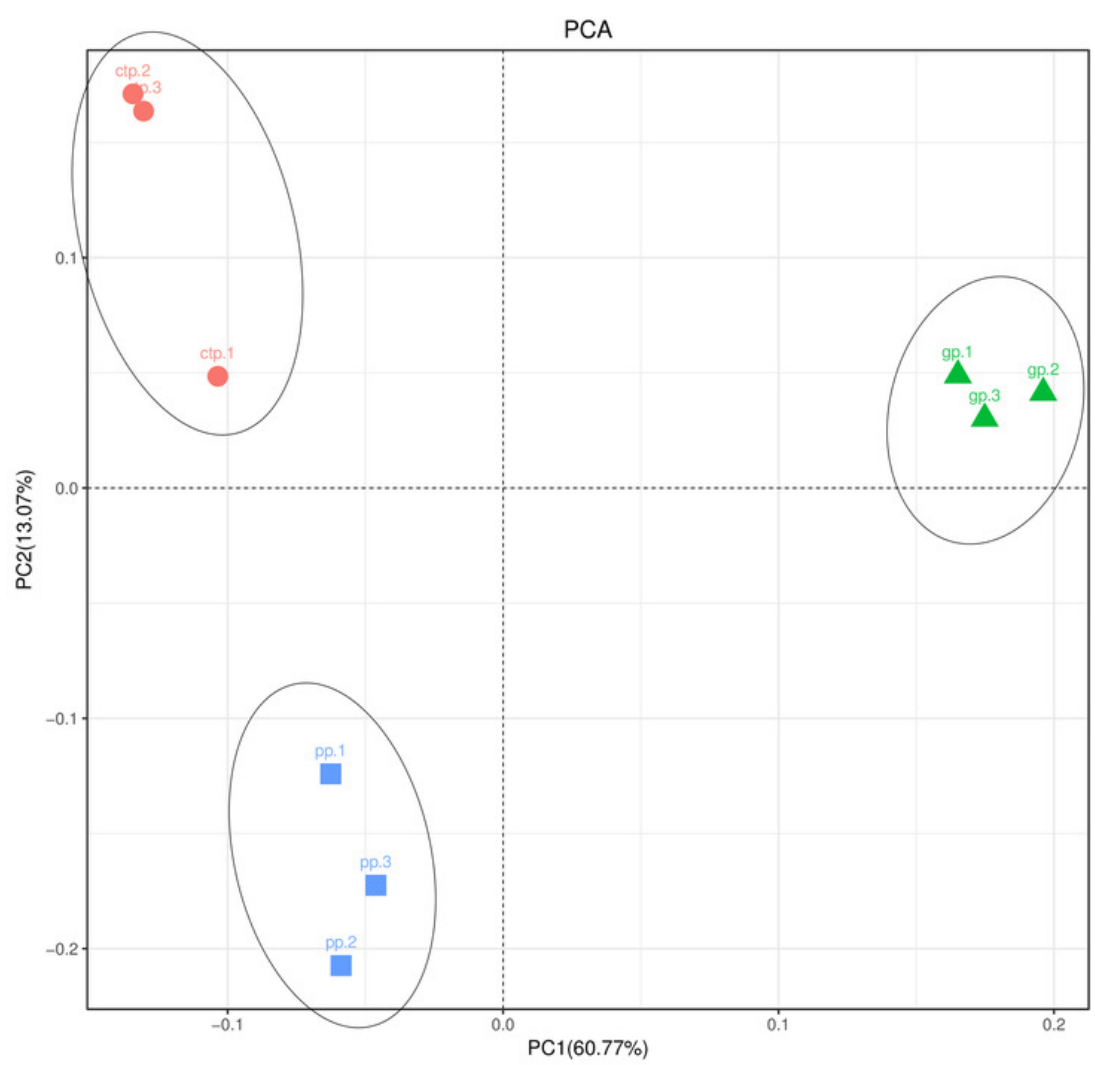

Group

$\Delta_{\mathrm{gp}}^{\mathrm{ctp}}$

pp

B

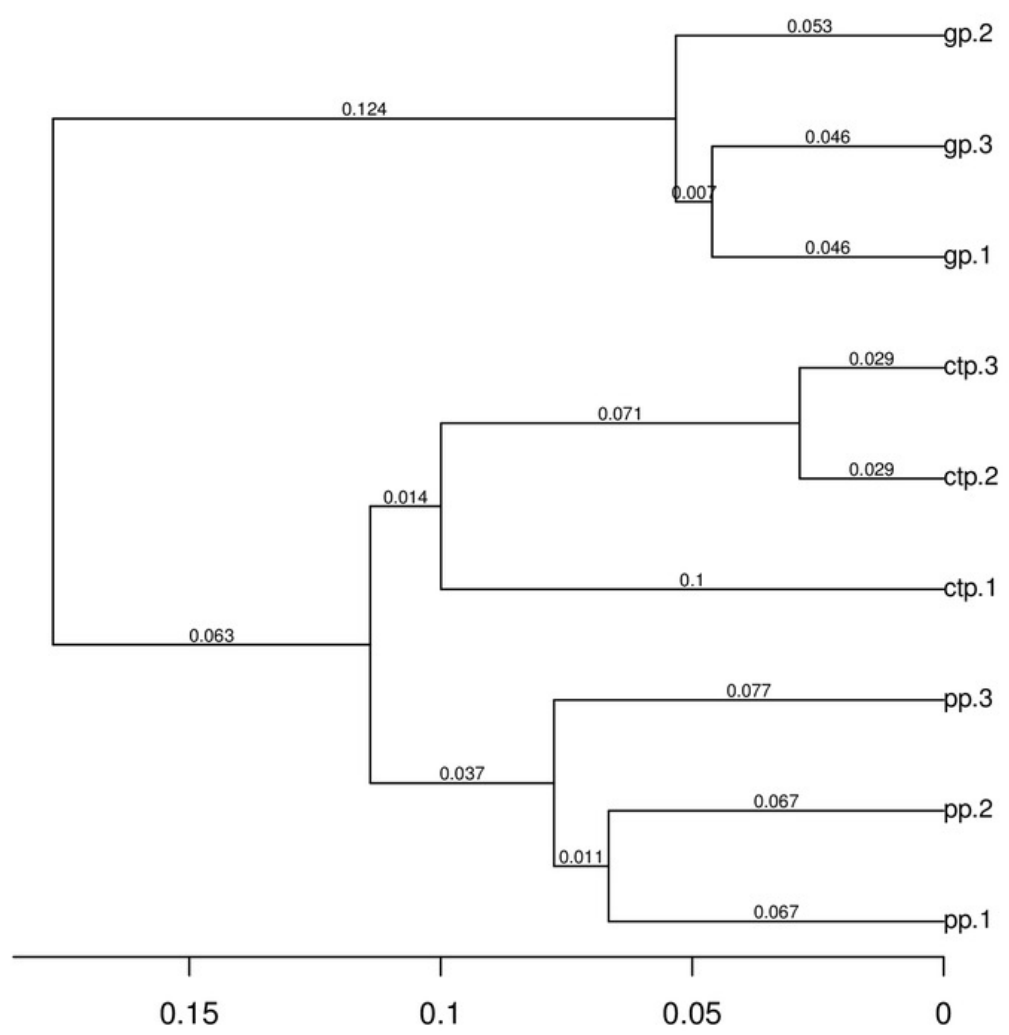


Figure 4

The inter-group difference classification unit display map based on the classification hierarchy tree.

The taxa hierarchy of all from gate to genus is shown from the inner to the outer circle. Node size represents the average relative abundance of OTUs; white nodes are OTUs with no significant difference between groups; red nodes are OTUs with high abundance in GP; green nodes are OTUs with high abundance in CTP; blue nodes are OTUs with high abundance in PP; letters are OTUs names with significant differences between groups.

Cladogram

$\mathbf{E}_{\infty}^{\infty}$

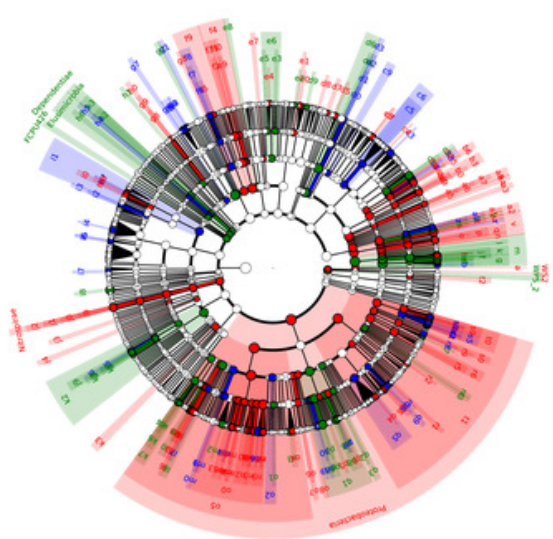

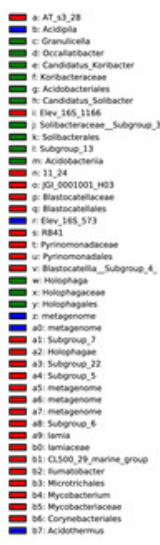
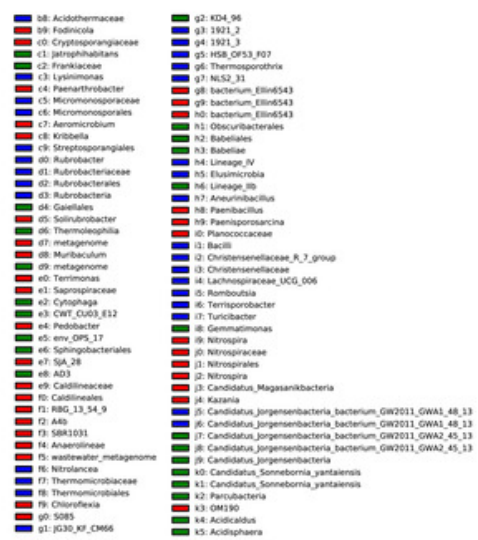

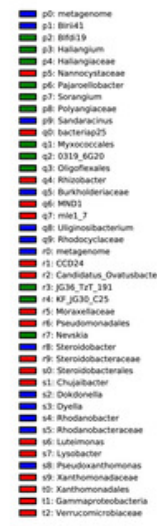


Figure 5

Heatmaps of the top 50 enriched functional groups.

The color code refers to gene abundance, with high predicted abundances (red) and low predicted (green). 


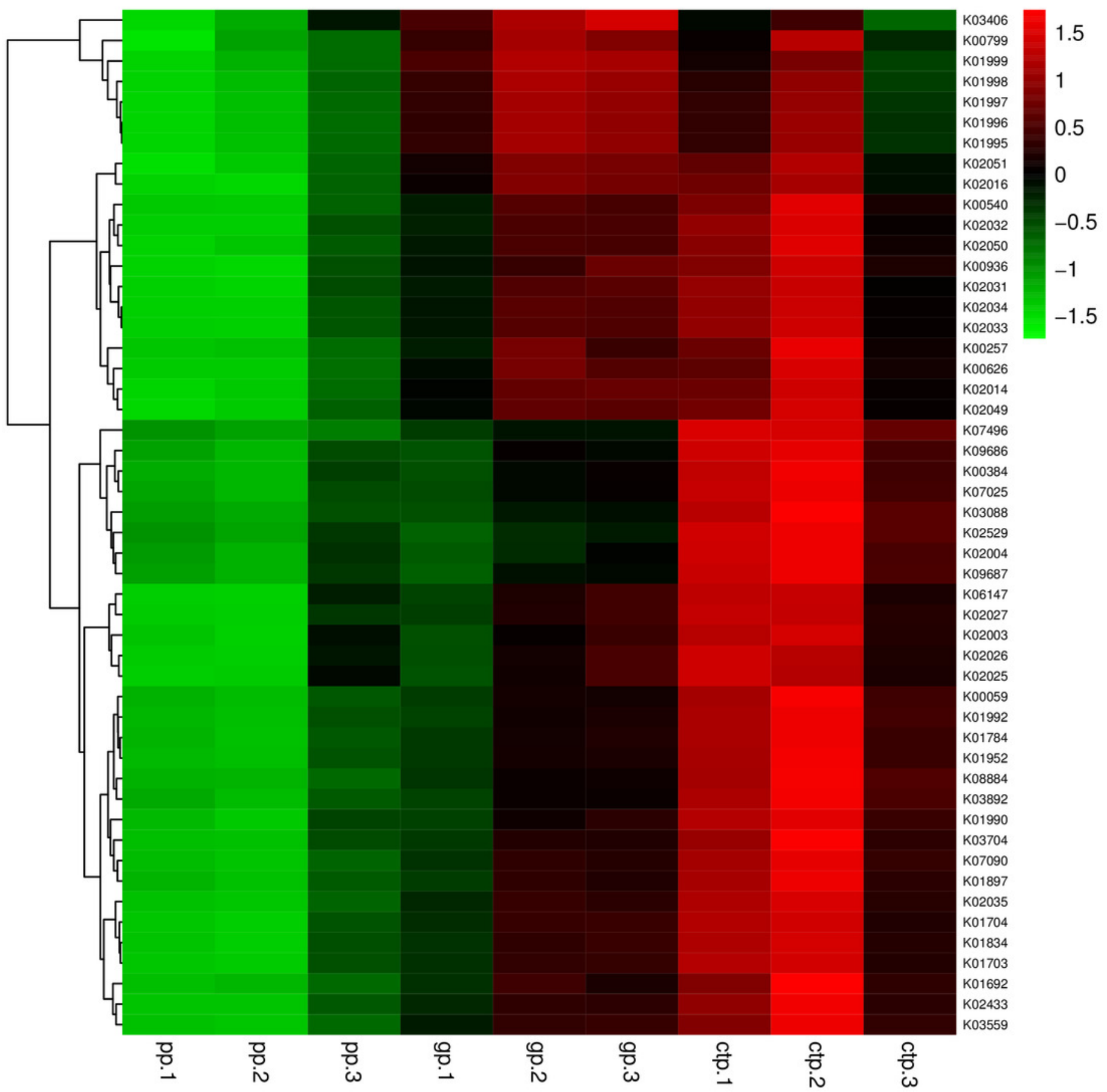




\section{Figure 6}

Venn diagram of OTUs for common functionally enriched soil bacteria among the three different shade shades.

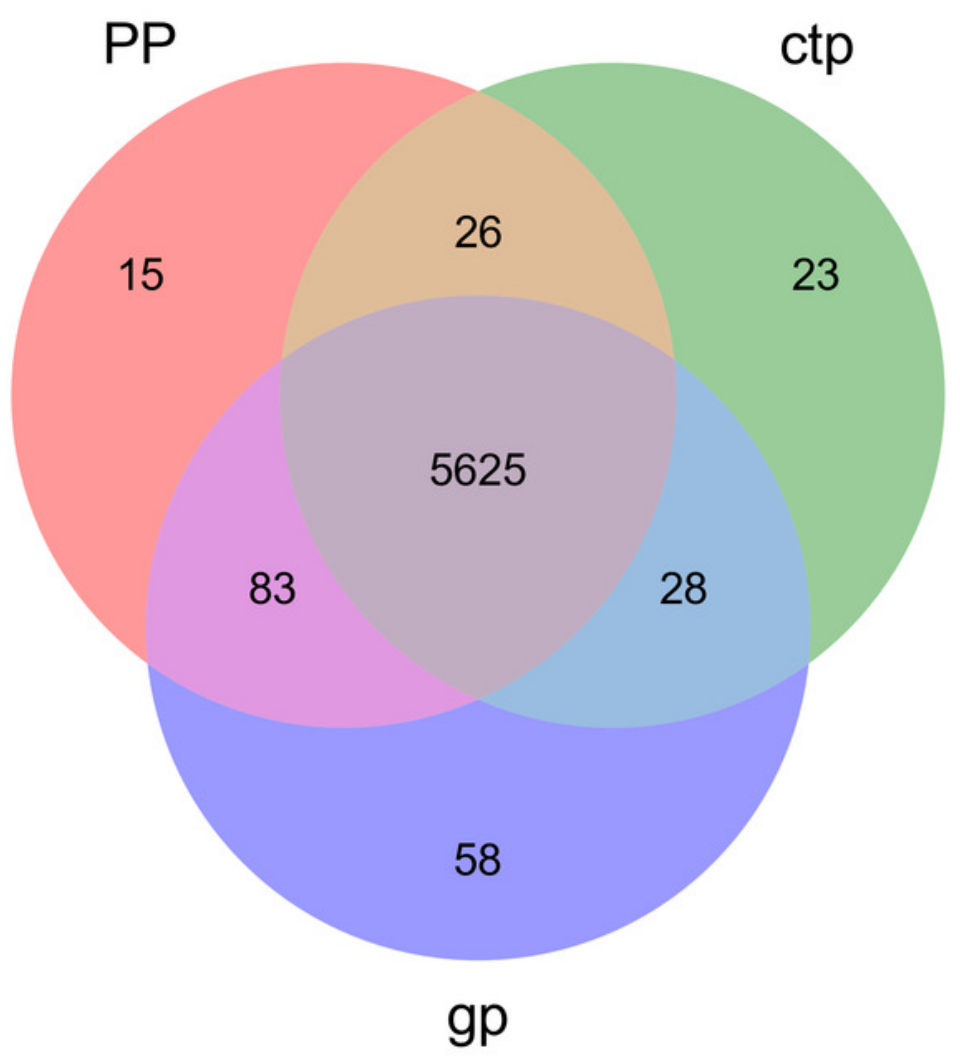


Figure 7

Correlation between soil bacterial diversity index and physicochemical properties under different shade sheds.

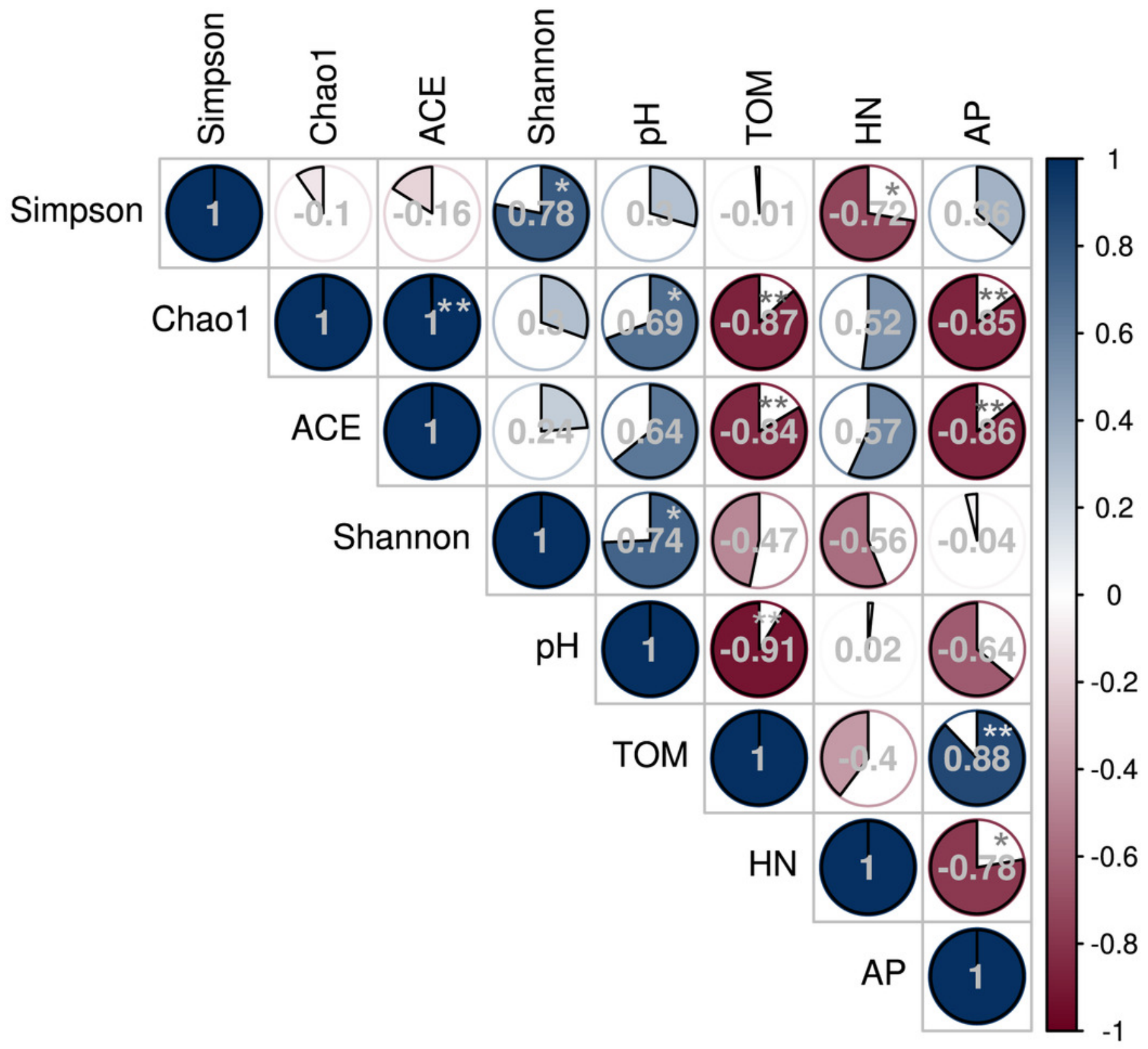


Figure 8

The RDA relationship between soil physicochemical and bacterial community composition.

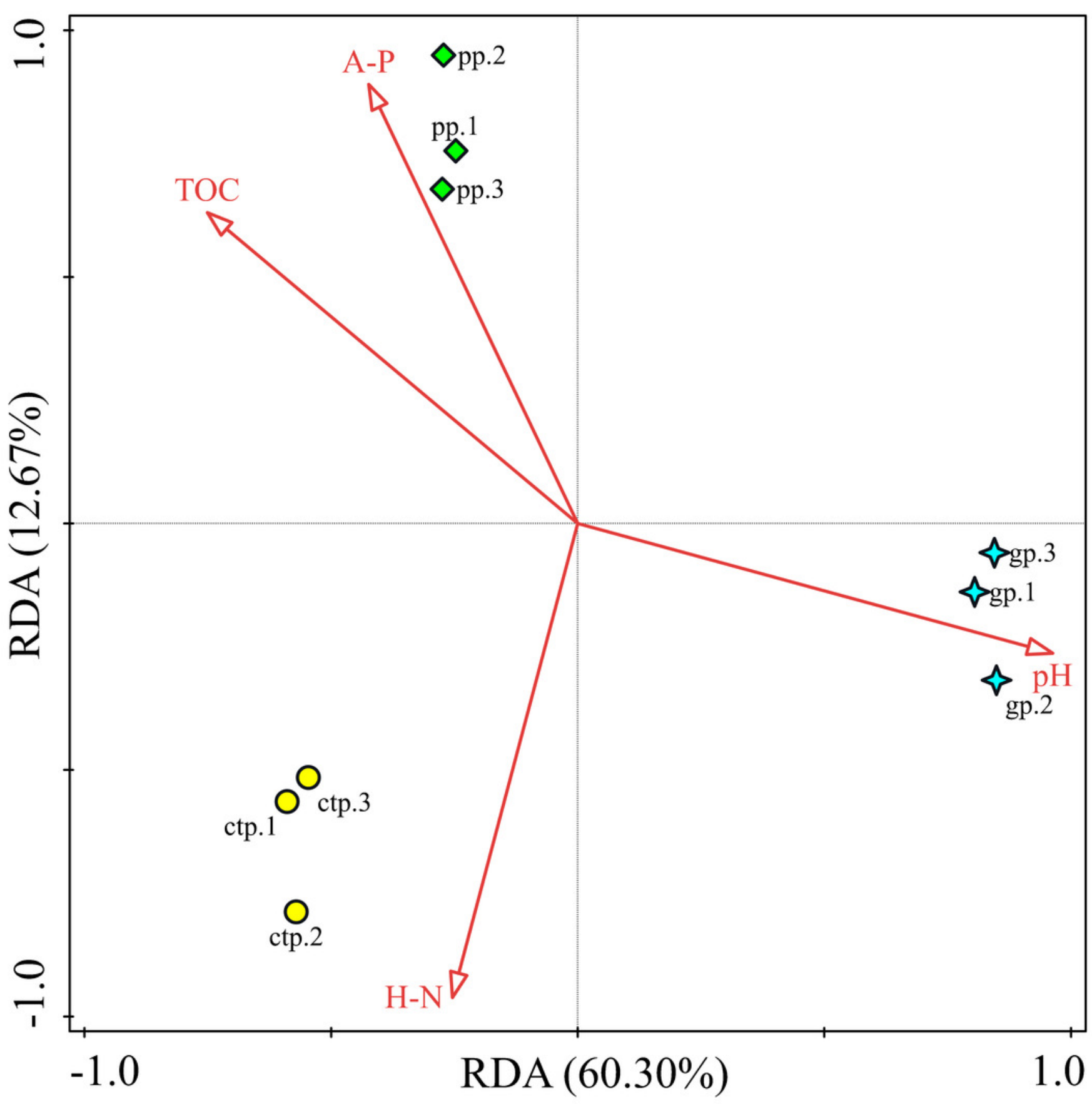

